\title{
Brain Injury MRI Simulator Based on Theoretical Models of Neuroanatomic Damage
}

\author{
L.M. González $\quad$ M. Luna $\quad$ A. García-Molina $\quad$ C. Cáceres J.M. Tormos and E. J. Gómez
}

\begin{abstract}
In order to improve the body of knowledge about brain injury impairment is essential to develop image database with different types of injuries. This paper proposes a new methodology to model three types of brain injury: stroke, tumor and traumatic brain injury; and implements a system to navigate among simulated MRI studies. These studies can be used on research studies, to validate new processing methods and as an educational tool, to show different types of brain injury and how they affect to neuroanatomic structures.
\end{abstract}

Keywords-Brain Injury, Traumatic Brain Injury (TBI), Tumor, Stroke, Feature Point, Magnetic Resonance Imaging (MRI), Simulator.

\section{INTRODUCTION}

Acquired brain injury (ABI) refers to any brain damage occurring after birth [1]. It usually causes certain damage to portions of the brain and result in a significant impairment of an individual's physical, cognitive and/or psychosocial functioning. Traumatic brain injury (TBI), stroke and brain tumors are the main causes of an ABI among others like infections or hypoxia. The consequence of $\mathrm{ABI}$ is the loss of capacity to execute their daily activities that lead to a dramatic change in the individual's daily life. One of the main challenges of neuroimaging is the development of incremental databases to link brain injury features with clinical outcome.

Over the last years, different research groups have developed open databases. Some approaches are 'The Whole Brain Atlas'(WBA) [2], or 'Internet Brain Segmentation Repository'(IBSR) [3]. However, we do not detect injured neuroanatomic databases simulating different types of brain injury. In order to improve the body of knowledge about brain injury impairment is essential to develop image database with different types of injuries. This kind of database permits to simulate injuries on healthy images. These simulated imaging studies improve medical educational and research procedures.

As an educational tool permits to simulate different types of brain injuries and to study the alterations suffering from neuroanatomic structures.

As a research tool is a potential tool to obtain controlled imaging studies of diverse brain injuries. The validation of methods to label neuroanatomic structures in neuroimaging studies [4] requires a huge amount of MRI studies and clinical effort to obtain the clinical assessment.

This paper proposes a methodology for modeling three types of brain injury: stroke, tumor and traumatic brain injury; and it implements a system to simulate those different types of brain injuries.

The remainder of this paper is organized as follows: section 2 reviews the different types of brain injury, section 3 explains the injured model used on each case, section 4 explains the dataset used, section 5 describes obtained results and finally section 6 discusses the results and explains the conclusions.

\section{TYPES OF BRAIN INJURY}

ABI neuroimaging presents different morphologies depending on the physiological mechanisms causing the injury. This paper considers three types of brain injury: brain tumor, stroke and traumatic brain injury (TBI). These kinds of brain injury are the most common ones.

\section{A. Brain Tumor}

A brain tumor is defined as an intracranial solid neoplasm within the brain [5]. In T1-MRI, as shown in Fig. 1 a tumor can be considered as an amorphous mass of variable size and location. Mostly, brain tumors present evident intensity imaging characteristics. Depending on the acquisition sequence of the MR, the tumor will be a hiperintensity or a hipointensity area. 


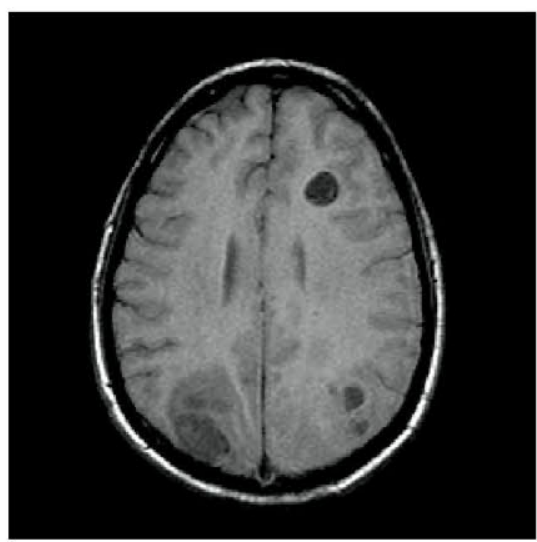

Fig. 1 Axial slice showing a sarcoma tumor T1-MRI

\section{B. Stroke}

A stroke or cerebrovascular accident is caused by an interruption of the blood flow in a region of the brain [6]. A stroke can be hemorrhagic or ischemic. A hemorrhagic stroke presents a diffuse area which is caused by bleeding or clotting (shown in Fig. 2). However, an ischemic stroke causes a necrosis on the infarction area.

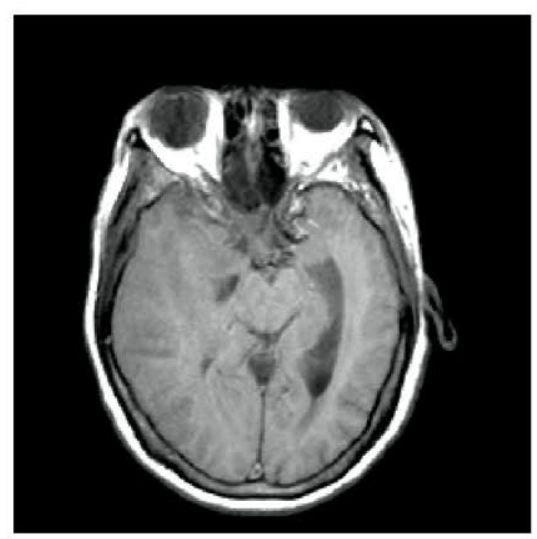

Fig. 2 Axial slice of a stroke

\section{Traumatic Brain Injury}

Traumatic Brain Injury (TBI) occurs when an external force traumatically injures the brain [7]. The trauma can be caused by a direct impact or by acceleration-deceleration process. MRI shows a TBI as a drift of the structures around the brain region affected by the impact (shown in Fig. 3) and on the opposite side of the brain, the brain area is smooth as a result of mass compression against the intracranial tissue.

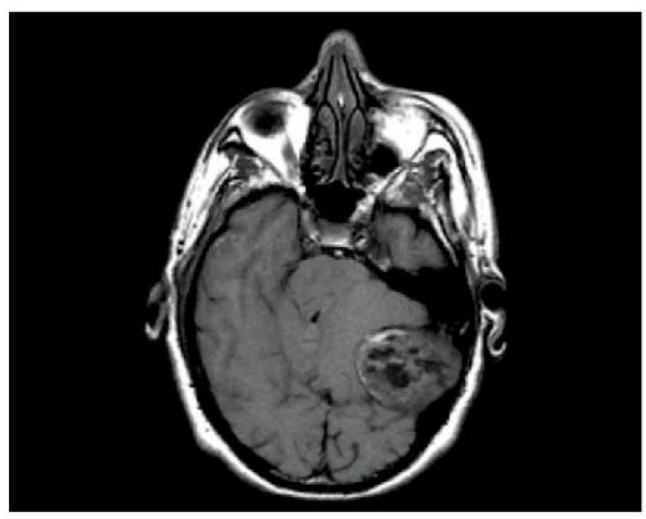

Fig. 3 Traumatic Brain Injury

\section{METHODS}

This section describes the methodology followed to model each type of brain injury. Firstly, the model of a brain tumor is described. Then, the model of a stroke is explained and finally, TBI model is characterized.

\section{A. Brain Tumor}

A brain tumor is considered to present a regular morphology. This paper considers a tumor as a spheroid that can appear in any location with random size. When a tumor is presented, not only an ellipsoid appears on the image but also neuroanatomic structures surrounding this area are moved.

Figure 4 shows a diagram of a simulated brain tumor imaging.
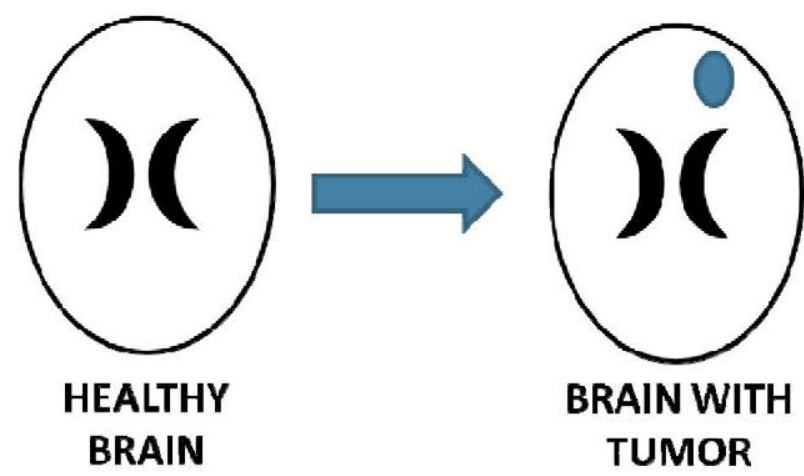

Fig. 4 Simulation of a brain tumor

As mentioned before, the proposed model generates a spheroid of variable size and random location. The volume defining the tumor is set as in Eq. 1(1). 


$$
S=\sqrt{\frac{\left(x-\text { tumor }_{x}\right)^{2}}{\text { tumor }_{x}}+\frac{\left(y-\text { tumor }_{y}\right)^{2}}{\text { tumor }_{y}}+\frac{\left(z-\text { tumor }_{z}\right)^{2}}{\text { tumor }_{z}}} \leq 1
$$

where tumor $_{x y z}$ is the radius of the tumor in the sagittal, coronal and axial plane. This parameter defines the location of the tumor within the brain volume. Finally, the image is smoothed by a Gaussian filter in order to achieve a non-uniform intensity area.

\section{B. Stroke}

In order to simulate a stroke brain a focus is defined. This focus represents where the blood supply is altered. As a consequence of the interruption of the blood supply a surrounding area around the focus is also affected. This area is also defined in the model, as shown in Fig. 5.

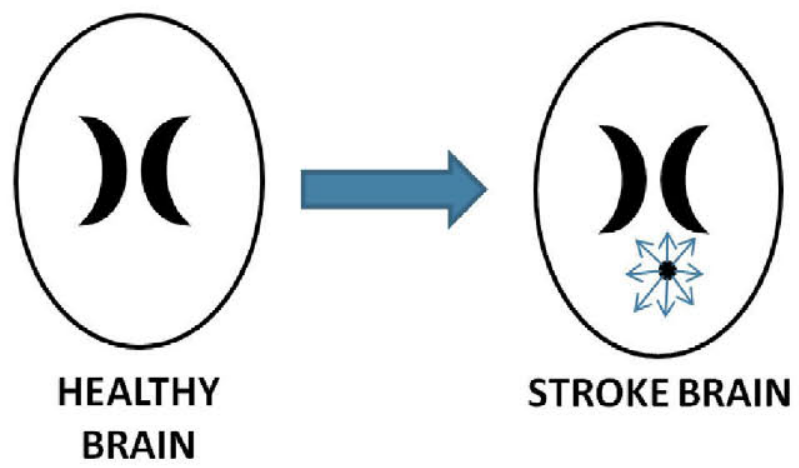

Fig. 5 Stroke

Then, this surrounding brain area is moved and intensity values are altered. The extension of the stroke and its flow direction are two parameters to be configured by the user.

\section{Traumatic Brain Injury}

A trauma is supposed to be originated by a hit on a side of the skull. This hit causes a neuroanatomic deformation. This deformation can be skull fracture or/and the displacement of brain tissues. The injured area is deformed due to different compression mechanism causing the disappearance of structures or/and a location change of them, as shown in Fig. 6.

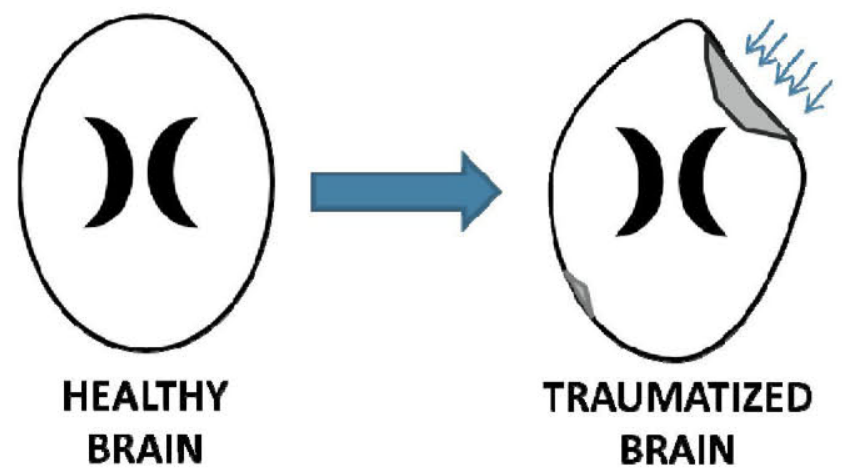

Fig. 6 Mechanism that produces a TBI

So as to simulate a TBI with a normal brain MRI. A mesh of points is defined and created over the brain region. This mesh computes the deformation on the selected brain region. The deformation is controlled with a local motion model by parameterized transformations based on B-splines [8]. The deformation is the product of $1 \mathrm{D}$ cubic B-splines (Eq. 2)

$$
\begin{aligned}
& T(x, y, z)= \\
& \sum_{l=0}^{3} \sum_{m=0}^{3} \sum_{n=0}^{3} B_{l}(u) B_{m}(v) B_{n}(w) \phi_{i+l, j+m, k+n}
\end{aligned}
$$

where $i=\left\lfloor x / n_{x}\right\rfloor-1, j=\left\lfloor y / n_{y} \mid-1, k=\left\lfloor z / n_{z}\right\rfloor-\right.$ $1, u=x / n_{x}-\left\lfloor x / n_{x}\right\rfloor, v=y / n_{y}-\left|y / n_{y}\right|, w=z / n_{z}-$ $\left\lfloor z / n_{z}\right\rfloor$ and $B_{l}$ is the $l$ th basis function of the B-spline.

A multiresolution approach is applying in order to increase the resolution of the control points of the mess. Control points are controlled by Eq. 3 (3):

$$
\begin{gathered}
\phi_{i, j, k}(\mathrm{~s}+1)= \\
\phi_{i, j, k}(\mathrm{~s})+\alpha * \sqrt{(\mathrm{i}+\mathrm{j}+\mathrm{k})-\left(\mathrm{c}_{\mathrm{i}}+\mathrm{c}_{\mathrm{j}}+\mathrm{c}_{\mathrm{k}}\right)}
\end{gathered}
$$

The updating of the mess is a function of position of each control point in relation with the core of the injury $c\left(c_{i}, c_{j}, c_{k}\right)$ and a parameter $\alpha$ defining the intensity of the movement. The deformation stops when the difference between the original volume and the deformed volume reach a determinate threshold.

\section{MATERIALS}

6 T1-MRI studies of healthy subjects have been used. These studies contain between 166 and 176 slices of $256 \times 256$ pixels.

Models and simulator system have been implemented on Matlab 2013a. 


\section{RESULTS}

Figure 7 shows an axial slice where a dark circle within the slice, represents a brain tumor. The tumor is the section of a spheroid that moves determinate neuroanatomical structures.

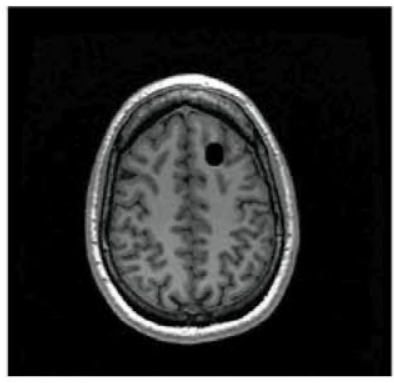

Fig. 7 Brain tumor model

Fig. 8 displays the difference between an original slice and the slice in a stroke case.

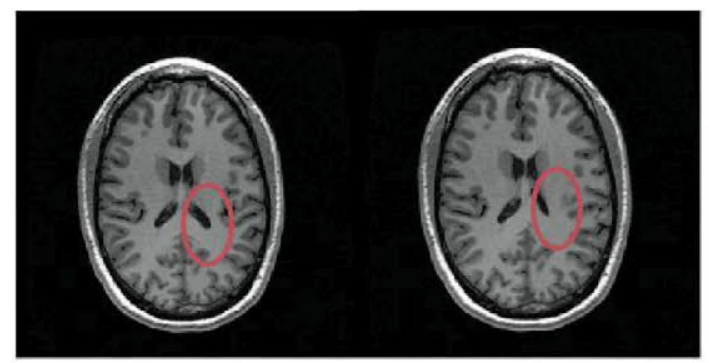

Fig. 8 Stroke simulation

The evolution of a TBI simulation is shown in Fig. 9. As observed, when increasing the force of deformation, more neuroanatomical structures are deformed.

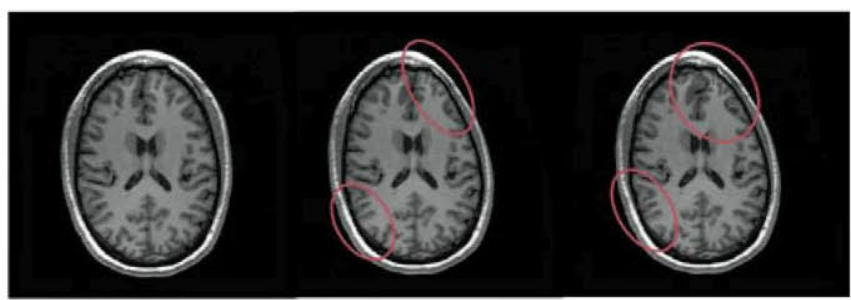

Fig. 9 TBI simulation

\section{CONCLUSIONS AND DISCUSSION}

We propose a system to simulate different types of brain injury on T1-MRI studies. This paper proposes a new methodology to model alteration in the image increasing the variability and size of our database of images.

The results shown permit to modify the structural information in order to simulate tumors, strokes or traumatic brain injury by deformed or translated health MRI studies.

The three proposed models will be improved in future papers by including more geometries, mechanical properties of different brain tissues.

\section{ACKNOWLEDGMENT}

This research has been partially founded by the Spanish Ministry of Economy and Finance (project TIN2012-38450, COGNITIO).

\section{REFERENCES}

1. Laxe S, Zasler N, Tschiesner U, López-Blazquez R, Tormos JM, Bernabeu M. (2011) ICF use to identify common problems on a TBI neurorehabilitation unit in Spain. NeuroRehabilitation, vol. 29, $\mathrm{n}^{\circ} 1$, pp. 99-110.

2. The Whole Brain Atlas(WBA) at http://www.med.harvard.edu/aanlib

3. Internet Brain Segmentation Repository(IBSR) webpage at http://www.cma.mgh.harvard.edu/ibsr/

4. Luna M, Gayá F, Sánchez P, Cáceres C, Pascual-Leone A, Tormos JM, Gómez EJ. Brain structures identification based on feature descriptor algorithm for Traumatic Brain Injury. International Conference on recent advances in neurorehabilitation (ICRAN), vol. 1 , pp.171-174, 2013

5. Herholz, Karl; Karl-Josef Langen, Christiaan Schiepers, James M. Mountz (2012). Brain Tumors. Semin Nucl Med. 42 (6): 356-70. doi 10.1053/j.semnuclmed.2012.06.001.

6. Kidwell CS, Chalela JA, Saver JL et al. (2004) Comparison of MRI and CT for detection of acute intracerebral hemorrhage. JAMA 292 (15): 1823-30. doi:10.1001/jama.292.15.1823

7. Maas AI, Stocchetti N, Bullock R (2008) Moderate and severe traumatic brain injury in adults. Lancet Neurology 7 (8): 728-41. doi:10.1016/S1474-4422(08)70164-9.

8. Rueckert, D., Sonoda, L., Hayes, C. et al. (1999) Nonrigid Registration Using Free-Form Deformations: Application to Breast MR Images. IEEE Trans. Med. Imag. 18(8): 712-21

Author: Luis Miguel González Rivas

Institute: Bioengineering and Telemedicine Centre, ETSI Telecomunicación, Universidad Politécnica de Madrid

Street: Avenida Complutense n ${ }^{\circ} 30$, "Ciudad Universitaria"

City: Madrid

Country: Spain

Email: lmgonzalez@gbt.tfo.upm.es 